\title{
Combination therapy in skin of color including injectables, laser, and light devices
}

\author{
Lark Guss, MD; ${ }^{1}$ Joanna G Bolton, MD, FAAD; ${ }^{2}$ and Sabrina Guillen Fabi, MD, FAAD, FAACS ${ }^{3,4}$
}

\section{Abstract}

With the rapid increase in patients seeking cosmetic treatments, the variation in responses of lightly pigmented skin versus darkly pigmented skin has become increasingly apparent. Despite extensive treatment options in patients with skin of color, there is a paucity of well-designed studies performed on this patient population. The lack of research is concerning, as it is well documented that patients with darker skin types are at an increased risk of adverse events when treated with many of the available modalities used in cosmetic procedures. Fortunately, by combining a variety of treatments, these risks may be abrogated, and combination treatments may be a promising regimen for a wide variety of cosmetic complaints. An overview and evaluation of the research of combination therapy in skin of color is presented.

Semin Cutan Med Surg 35:211-217 @ 2016 Frontline Medical Communications

$\mathrm{L}$ aser, light, and energy devices and a variety of injectable and topical products have revolutionized treatment of dyspigmentation, skin texture, and skin laxity over the past few decades. Combining treatment modalities to achieve better outcomes is becoming increasingly popular. Although these treatments were traditionally performed in patients with lighter skin types, the improved understanding of the effects of these treatments, particularly lasers, on pigmented skin has led to their increasing use in skin of color.

The increased baseline pigment of the epidermis and larger melanosomes more widely distributed throughout epidermal keratinocytes confer a greater risk of dyschromia following laser procedures using wavelengths that have melanin as a chromophore. ${ }^{1,2}$ Optimizing laser parameters, such as increasing pulse durations, longer wavelengths, and attached cooling devices, minimize the risk of adverse events. ${ }^{3,4}$ However, treatment of darkly pigmented patients continues to be associated with an increased risk of postinflammatory hyperpigmentation (PIH) and prolonged erythema. ${ }^{5}$

\footnotetext{
${ }^{1}$ Dermatology Resident, Department of Dermatology, Johns Hopkins University, Baltimore, Maryland.

${ }^{2}$ Cosmetic Dermatologic Surgery Fellow, Goldman, Butterwick, Groff, Fabi \& Wu, Cosmetic Laser Dermatology, San Diego, California.

${ }^{3}$ Associate, Goldman, Butterwick, Groff, Fabi \& Wu, Cosmetic Laser Dermatology, San Diego, California.

${ }^{4}$ Voluntary Assistant Clinical Professor in Medicine/Dermatology, University of California, San Diego, California.

Disclosures: The authors report no relevant conflicts of interest. Correspondence: Lark Guss, MD; Department of Dermatology; Johns Hopkins University; 601 N. Caroline St, 8th floor, Baltimore, Maryland 21287. Email: lgreenw5@jhu.edu
}

Thus, many recent studies have begun to investigate the benefits of combining procedures and treatments to maximize benefits while decreasing the risk of adverse events.

\section{Face}

\section{Melasma}

Melasma, an acquired disorder of hyperpigmentation, is one of the most common types of pigmented lesions found in patients with skin of color. ${ }^{6}$ Though its exact cause is unknown, melasma is exquisitely photosensitive and exacerbated by hormones. Traditional treatment has typically relied upon strict physical sunblock use in conjunction with a topical combination of tretinoin, hydroquinone, and a topical steroid. ${ }^{6}$ However, although a topical cream may lighten epidermal pigment, it does little to alleviate pigment centered in the dermal layer of the skin. Additionally, prolonged use of topical hydroquinone may cause paradoxical hyperpigmentation due to exogenous ochronosis, and irritation secondary to topical retinoids may induce skin irritation, increasing the risk of PIH.

Thus, lasers have been investigated for efficacy in treatment of melasma given their potential to reach deeper depths in the dermis. In order to bypass epidermal pigment, several studies have attempted pretreating with an ablative laser to remove the epidermis, thereby decreasing intervening epidermal pigment between a Q-switched (QS) laser and dermal pigment. Angsuwarangsee et al treated 6 Thai patients with an ultrapulse carbon dioxide $(\mathrm{CO} 2)$ ablative laser to one side of the face followed by a QS alexandrite 755-nm laser to the entire face. Six months following the procedure, the side of the face with combination treatment had significant lightening. There was no significant reduction of pigment on the side of the face treated with a QS alexandrite laser alone. Notably, when the 2 sides were compared directly, the difference in treatments did not reach statistical significance. Additionally, those patients with Type IV-V skin required a topical bleaching agent 3 months after their laser treatment to address their PIH. ${ }^{7}$ However, the relative success of the combination approach confirmed similar findings found using a pulsed $\mathrm{CO} 2$ ablative laser followed by a QS alexandrite laser. All 4 patients studied had improvement with the combination treatment, and no PIH was noted. ${ }^{8}$ A combination of intense pulsed light (IPL) followed by a QS 1064-nm Nd:YAG fractionated laser also had a significant lightening effect in patients with Type III-IV skin with no PIH noted. ${ }^{9}$ The authors hypothesized that the success of the treatment relied on the large action spectrum of the 2 lasers, with IPL covering epidermal pigment and dermal pigment covered by the QS Nd:YAG.

Low fluence QS Nd:YAG alone can successfully treat melasma; however, numerous weekly treatments are needed, and postprocedure hypopigmentation has been documented. ${ }^{10,11}$ However, when treated with 5 sessions of IPL immediately following low fluence QS Nd:YAG, 14 out of 18 patients rated their response as good to 


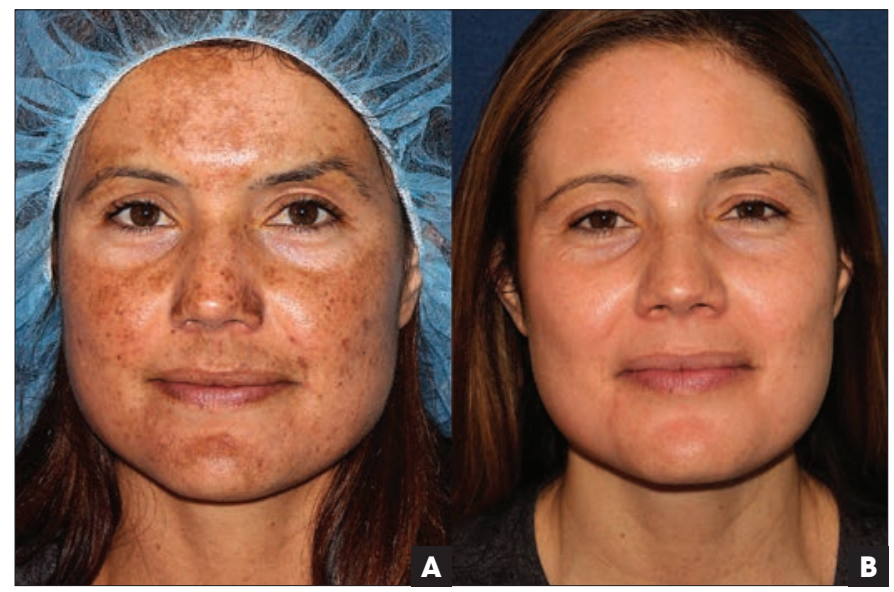

FIGURE 1. (A) A 35-year-old skin type III female with severe melasma treated over the course of 1 year with 4 consecutive IPL treatments spaced 1 month apart followed by 8 consecutive 755-nm picosecond laser treatments spaced 1 month apart in combination with topical product and sun protective measures. A ninth 755-nm picosecond treatment was performed at the 12-month follow-up for maintenance. The photo in (B) was taken an additional 12 months after the ninth laser treatment (2 years after baseline). (A) Baseline; (B) 12 months status post ninth laser treatment. Used with permission: Mitchel P Goldman, MD.

excellent, and only 1 patient developed guttate hypomelanosis. ${ }^{12}$ Treatment numbers with low fluence QS Nd:YAG may also be lowered by pretreating initially with microdermabrasion. When 27 women were treated with microdermabrasion followed immediately by QS Nd:YAG, the mean number of monthly treatments was only 2.6 , and $85 \%$ of women experienced a $75 \%$ or greater improvement in their melasma. ${ }^{13}$ Additionally, a more recent splitface study compared a QS 1064-nm Nd:YAG laser with a QS 755$\mathrm{nm}$ alexandrite laser using very low fluences of only $1-2 \mathrm{~J} / \mathrm{cm}^{2}$ for the treatment of melasma. In 18 patients with types III and IV skin, pigmentation improved equally well on both sides of the face after 6 weekly treatments, and there were no adverse events aside from minimal transient erythema. ${ }^{14}$ Thus, though using very low fluences requires multiple treatments, it does appear to minimize the risk of dyschromia while still maintaining efficacy.

Perhaps because laser and light treatments can be manipulated to very narrowly target melanocytes in different layers of the skin, combinations of these treatments appear to work better than combination treatments of lasers with topical treatments such as chemical peels. When $30 \%$ glycolic acid peels were added to low fluence QS Nd:YAG laser sessions, 15 male patients with melasma were found to have only transient improvement, and 1 patient developed permanent guttate hypopigmentation. ${ }^{15}$ Thus, combination laser treatment does appear to be a viable option in patients with melasma unresponsive to topical therapy alone.

In our practice, a combination of IPL (560-nm filter) and 755nm picosecond laser (Picosure, Cynosure, Inc, Westford, Massachusetts) or 755-nm QS laser (Accolade, Cynosure, Inc, Westford, Massachusetts) in conjunction with topical treatments has been utilized to successfully treat a skin type III patient (Figure 1). The 35-year-old female suffered from severe melasma refractory to conventional topical therapy and sun protective measures, and

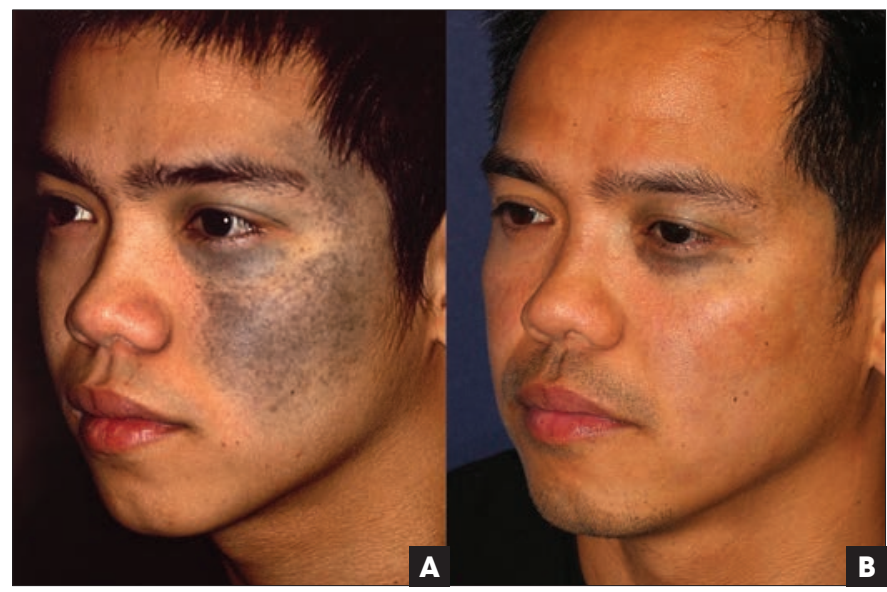

FIGURE 2. Figure 2. A 37-year-old skin type IV male with Nevus of Ota. (A) Baseline; (B) Status post QS alexandrite and picosecond 1064-nm and 755-nm treatments over a 17-year period. Treatment remains ongoing for lower eyelid with picosecond wavelengths. Used with permission: Mitchel P Goldman, MD.

her condition worsened following glycolic and Jessner peels obtained prior to consultation. Over the course of a year, she underwent 4 consecutive IPL treatments spaced 1 month apart followed by 8 consecutive treatments with the $755-\mathrm{nm}$ picosecond laser at 1 -month intervals. The fluence ranged from 16 to $18 \mathrm{~J} / \mathrm{cm}^{2}$ for the IPL treatments, double-pulsed with a $3-\mathrm{mm}$ pulse duration and 30 ms pulse delay. For the 755-nm picosecond treatments, the spot size ranged from $2.5 \mathrm{~mm}$ to $6.0 \mathrm{~mm}$ with a fluence range of 0.71 to $4.07 \mathrm{~J} / \mathrm{cm}^{2}$. A ninth $755-\mathrm{nm}$ picosecond laser treatment was performed at her 12-month follow-up appointment for maintenance. Throughout her treatment cycle, regular use of a topical skin lightening product (Lytera, SkinMedica Inc, Carlsbad, California) and strict sun protection measures were practiced. Results have been maintained for greater than 1 year since last treatment.

\section{Nevus of Ota}

Nevus of Ota is a dermal melanocytic nevus most often seen in Asian patients as a dark-blue pigmented patch in the trigeminal region. ${ }^{16}$ Numerous QS lasers of varying wavelengths have been shown to be efficacious, though all require numerous treatments. ${ }^{17-19}$ Additionally, swelling, crusting, and hypo- and hyperpigmentation are well-documented adverse events following treatment. ${ }^{17,20}$ In comparing a QS alexandrite, QS Nd:YAG, and a regimen alternating between the 2 lasers, the combination treatment group appeared to have a greater degree of clearing, though there was no significant difference when accounting for the number of treatment sessions. ${ }^{16}$ Hypopigmentation was noted to occur in all 3 groups and was highest in the combination treatment group at $38 \% .{ }^{16}$ Thus, for treatment of Nevus of Ota, evidence does not support alternating between 2 types of QS lasers.

With the recent usage of picosecond lasers, there is another tool available for treating Nevus of Ota. The patient in Figure 2 initially presented to our practice in 1999 and received a series of QS alexandrite treatments over 10 years. During this initial set of treatments, the lower eyelid was not specifically targeted, because internal eye shields were not utilized. Following a 5-year lapse in follow-up, the patient presented desiring additional treatment for 
remaining pigment in the previously treated areas as well as the pigment on the lower eyelid. To date, 2 treatments with the 1064$\mathrm{nm}$ picosecond laser (PicoWay, Syneron Candela, Irvine, California; fluence ranging from 3.0-3.2 J/cm2, 4-mm spot size, 3-4 Hz) and 1 treatment with the $755-\mathrm{nm}$ picosecond laser (fluence of 2.08 $\mathrm{J} / \mathrm{cm}^{2}, 3.5-\mathrm{mm}$ spot size, $10 \mathrm{~Hz}$ ) have been performed following placement of internal metal eye shields. At this time, the patient remains in follow-up every 6-8 weeks with additional picosecond laser treatments planned to target remaining pigment.

Nevus of Ota is rarely the only etiology of dyspigmentation on the face. Patients often have concurrent pigmentary lesions, such as melasma, pigmented seborrheic keratoses, ephelides, or lentigines, and thus may be classified as having complex dyspigmentation. ${ }^{21}$ Park et al attempted to combine global photorejuvenation with targeted pigment therapy by beginning with IPL therapy and following with 694-nm QS ruby laser in 25 Korean patients with at least 2 pigmentary disorders, and 4 of whom had acquired Nevus of Ota; $60 \%$ experienced a 75\%-100\% improvement as assessed by 2 independent dermatologists. Three patients had transient PIH that resolved with subsequent IPL treatments, and 1 patient had $\mathrm{PIH} .{ }^{21}$ As the incidence of PIH can be high regardless of the laser used, the authors commonly perform a test spot to assess skin reaction and clearance from the treatment prior to treating the entire area.

\section{Acne scars}

Severe acne can have a significant deleterious impact on quality of life. Without adequate treatment, the scarring and pigmentary changes may persist indefinitely despite clearance of the acne itself. Therefore, acne and acne scarring are common complaints in patients seeking dermatologic treatment. Several types of scars may develop after acne through dermal fibrosis or pigmentary alteration, and treatment of the scars is challenging, as more hyperpigmentation or scarring may occur. Additionally, multiple types of lesions may be contributing to a patient's undesired appearance. For example, it is not uncommon to have both an inflammatory and scarring component at the same time. Thus, severe acne and acne scarring may be better treated with multiple modalities able to target the underlying lesions most effectively.

Though isotretinoin is often effective and first line, many patients cannot tolerate the excessive dryness or develop one of the rarer, severe side effects. Topical 5-aminolaevulinic acid-photodynamic therapy (ALA-PDT) has long been used for treatment of actinic keratoses but has also shown efficacy for treatment of acne and is safe in patients with pigmented skin. ${ }^{22,23}$ By using a combination of both ALA-PDT treatment with an ablative fractional Er:YAG 2940-nm laser in 40 patients with skin types III-IV, Yin et al showed complete resolution of all active acne lesions after 4-5 monthly sessions and no new scars. Six months following the last treatment, $80 \%$ of subjects' scars improved by more than $50 \%$, and $25 \%$ of scars improved more than $75 \% .{ }^{24} \mathrm{~A}$ third of patients developed transient PIH, though all improved by 6 months.

In our practice, we routinely use a combination of ALA-PDT and pulsed-dye laser (PDL) for treatment of active acne and erythematous acne scars. In certain cases, IPL is also incorporated into the regimen. ALA is applied to the face after extractions and exfoliation with a vibrating microdermabrasion system (Vibraderm, Grand Prairie, Texas) are performed. It is then degreased

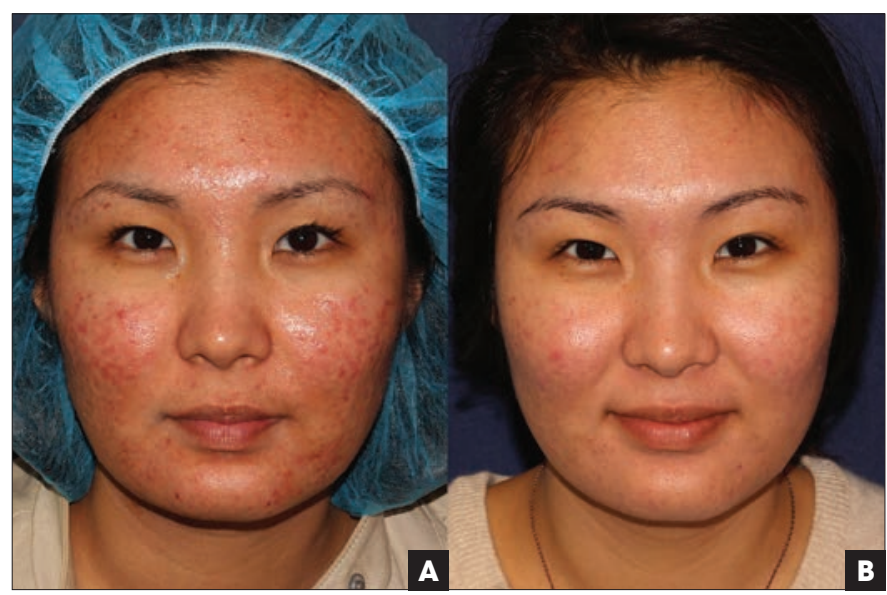

FIGURE 3. A 22-year-old skin type IV female with active acne and numerous erythematous acne scars. (A) Baseline; (B) 3 months status post combination PDT utilizing red and blue light sources, PDL laser, and use of oral spironolactone and topical benzoyl peroxide.

with an acetone wash. Figure 3 demonstrates a 22-year-old skin type IV female treated with subpurpuric full-face PDL to activate the photosensitizer followed by simultaneous illumination with a blue light (Blu-U, DUSA Pharmaceuticals, Inc, Wilmington, Massachusetts) source positioned 25 to $50 \mathrm{~mm}$ from the skin for an illumination period of 16 minutes 40 seconds, light dose 10 $\mathrm{J} / \mathrm{cm}^{2}$, and a red light source (Aktilite CL 128, PhotoCure ASA, Oslo, Norway) positioned 50 to $80 \mathrm{~mm}$ from the skin for a total of 8 minutes, 49 seconds at a standardized fluence of $37 \mathrm{~J} / \mathrm{cm}^{2}$. Incubation with ALA was 3 hours prior to treatment. The patient was prescribed oral spironolactone (50 $\mathrm{mg}$ twice daily) and instructed to use topical benzoyl peroxide as needed for spot treatment, as she could not tolerate daily treatment over her entire face. At the 3 -month follow-up, greater than $75 \%$ clearance was noted. The patient has continued her oral and topical medications with additional PDL treatments planned as needed, and fractional CO2 resurfacing has been recommended as part of her combination treatment plan.

In a pilot study in which 22 subjects (Fitzpatrick Skin Types $\mathrm{IV}-\mathrm{V}=9 / 22$ ) received 3 treatments 14 days apart with microfocused ultrasound (MFU-V) using the $1.5-\mathrm{mm}, 10-\mathrm{MHz}$ (300 total lines to the entire face) and 1- mm, 10- MHz (420 lines to the entire face) depth transducers, a significant decrease in sebum, as measured by a sebumeter (Courage-Khazaka, Köln, Germany), was noted over the forehead, cheeks, and chin 60 days post treatment. Eighty percent of subjects had a decrease in total acne lesion count at 60 days, and $100 \%$ of subjects showed a decrease at 180 days after the last treatment. It is hypothesized that, with the use of 1.5-mm and 1-mm depth probes, sebaceous glands are targeted for coagulation while bypassing the basal layer. ${ }^{25}$ Data from this pilot trial suggest that MFU-V could prove to be a promising novel treatment option to improve acne clearance in those with moderate to severe inflammatory acne. ${ }^{26}$ An additional study found that 20 patients ( $75 \%$ of whom had skin type III or above) all noted improvement of their atrophic acne scars following 3 monthly sessions of MFU-V. ${ }^{27}$

In a Chinese study, 37 patients with both atrophic scars and in- 
flammatory lesions were treated with 4-6 successive IPL sessions (fluence ranging from $14-18 \mathrm{~J} / \mathrm{cm}^{2}$, pulse width $3.0-4.0 \mathrm{~ms}$, and delay time of 25-40 ms) followed by 2 sessions of fractional ablative $\mathrm{CO} 2$ laser. Patients were treated with 2 passes of the $\mathrm{CO} 2$ laser, the first with a spot size of $0.12 \mathrm{~mm}$, scan size $10 \times 10 \mathrm{~mm}$, repetition rate of 300-400 Hz, pulse energy $10-15 \mathrm{~mJ}$, and density of $5 \%$ (Deep FX; Lumenis Ltd, Santa Clara, California). The second pass used a spot size of $1.3 \mathrm{~mm}$, scan size of 7-9 mm, repetition rate of 300-350 Hz, pulse energy of 90-150 mJ, and density setting of 3-5 (Active FX; Lumenis Ltd, Santa Clara, California). Though the inflammatory lesions decreased significantly following IPL, scarring improved only following the $\mathrm{CO} 2$ laser treatments. PIH was seen in almost $40 \%$ of patients following the fractional $\mathrm{CO} 2$ sessions, though all resolved by 3 months. ${ }^{28}$

Though fractional resurfacing is a well-documented treatment of acne scars, numerous treatment sessions are often required to induce significant improvement. ${ }^{29}$ Additionally, PIH and scarring are known side effects. As PIH is much more likely to occur in patients of darker skin types, lower density settings and/or longer treatment intervals are often required. ${ }^{30}$ Thus, combination treatments have been sought to limit the side effects of ablative resurfacing and to enhance the therapeutic effect.

Radiofrequency (RF) treatment devices use 1 or 2 electrodes to heat the dermis and cause collagen contraction and skin tightening. ${ }^{31}$ Thermal damage also recruits fibroblasts to the areas of injury, which increase collagen synthesis. ${ }^{31}$ When RF devices are combined with nonablative lasers, atrophic acne scars have been shown to improve by $60 \%-72 \% .^{32,33}$

Additionally, high-intensity bipolar RF (Infini, Lutronic Inc, Burlington, Massachusetts) targets the dermis in a highly focused manner by varying the depth of the microneedles on the device. ${ }^{34}$ This technique bypasses the epidermis and directly heats the dermis to maximize remodeling beneath acne scars. This protective epidermal effect is particularly beneficial in pigmented skin while maintaining efficacy deeper in the tissue. ${ }^{34}$

Combination treatments utilizing topical applications following laser procedures have also been explored to address scarring. In 13 patients with skin types III-IV, facial skin was treated with the conditioned medium of adipose-derived stem cells (ADSCs) in the hopes that healing of laser-damaged skin would be accelerated. ${ }^{35}$ The numerous growth factors secreted by ADSCs into their surrounding medium, such as basic fibroblast growth factor, keratinocyte growth factor, transforming growth factor, and vascular endothelial growth factor, may stimulate migration of fibroblasts into the dermis in addition to dermal collagen synthesis. ${ }^{36}$ Following 2 passes of a fractional CO2 laser, 1 side of the face was treated with the conditioned medium of ADSCs. Three monthly sessions were performed. One month following the last treatment, participant satisfaction and objective clinical assessment were both significantly improved on the ADSC-medium treated side. No persistent adverse events were noted, and there was no significant difference in pain or erythema between the 2 sides. $^{45}$ Yet, there was reduced hyperpigmentation using the ADSC medium.

\section{Facial photodamage and rejuvenation}

The conditioned medium of ADSCs has also been used immediately following fractional $\mathrm{CO} 2$ laser when treating facial photo-

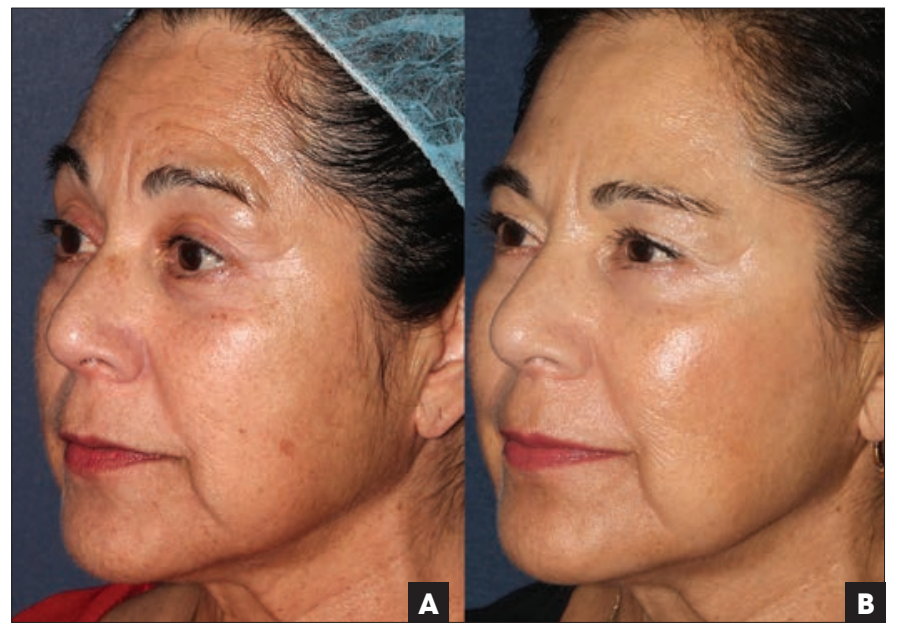

FIGURE 4. A 5-year-old skin type IV female with facial photodamage. (A) Baseline; (B) 12 months status post soft tissue filler for the cheeks and chin, and a QS 755-nm alexandrite laser to target individual pigmented macular seborrheic keratoses, immediately followed by a 1927-nm fractionated thulium laser to the full face, followed by neuromodulator injection 2 weeks later.

damage in skin types III-IV. ${ }^{37}$ Because the laser forms microscopic holes in the skin, drug delivery is hypothesized to be significantly enhanced. Thus, the growth factors in the ADSC medium should be able to more efficiently recruit dermal fibroblasts and increase dermal collagen. In fact, histologic analysis of the subjects' skin showed increased dermal collagen density and more ordered collagen than the facial side treated with laser alone. ${ }^{37}$

For noninvasive treatment of facial wrinkles, a combination of optical energy with RF or heat energy are thought to work synergistically with each other. First, the optical energy specifically heats a target, which then further concentrates the heat energy administered by the RF device. ${ }^{38}$ Twenty-three patients were treated for facial and neck wrinkles with up to 3 sessions of a combination diode laser and RF system (Polaris, Syneron Medical Ltd, Yokneam, Israel), after which $30 \%$ experienced at least a $50 \%$ improvement and $66 \%$ noted at least a $25 \%$ improvement, with no reports of pigmentary change. ${ }^{39}$ Patients had skin phototypes ranging from II to IV, though there were no notable differences in efficacy based upon skin type.

In our practice, fractionated ablative and nonablative lasers are routinely used for rejuvenation of facial photodamage in skin types III-V in combination with other lasers, injectable fillers and neuromodulators, and medical grade lightening products. The skin type IV patient in Figure 4 presented as an anti-aging consult, most concerned about the lentigines, volume loss, and overall photodamage on her face. On the same day, the patient underwent treatment with a soft tissue filler for the cheeks and chin (Radiesse, Merz, Raleigh, North Carolina) and a QS 755-nm alexandrite laser to target individual pigmented macular seborrheic keratoses (3-mm spot size, 7 $\mathrm{mJ} / \mathrm{cm}^{2}, 10 \mathrm{~Hz}$ ), immediately followed by a $1927-\mathrm{nm}$ fractionated thulium laser to the full face (Fraxel Restore, Solta Medical Inc., Hayward California) (20 mJ, 50\% coverage [treatment level 7] delivered over 8 passes). Over the course of the year, she also had neuromodulation (Botox, Allergan, Irvine, California) and started routine use of a skin lightening regimen (SkinMedica Inc, Carls- 
bad, California). Future treatment with additional filler and MFU$\mathrm{V}$ for skin tightening has been recommended as part of her ongoing combination treatment plan.

\section{Neck}

\section{Skin laxity}

Due to ultraviolet radiation damage and chronological aging, dermal collagen and elastin gradually diminish, resulting in skin laxity, which may be particularly pronounced over the neck. ${ }^{40}$ For superficial skin tightening, fractionated ablative $\mathrm{CO} 2$ laser has been shown to improve neck laxity by an average of $57 \%$ at 2 months. ${ }^{41}$ However, as noted above, even fractional $\mathrm{CO} 2$ treatment of patients with pigmented skin carries a high risk of PIH. ${ }^{28}$ Newer devices utilizing radiowave or ultrasound technology have become increasingly popular for treatment of off-face skin laxity. ${ }^{42}$ As neither radio nor ultrasound waves target pigment, their use in patients with darker skin types typically carries no greater risk than treatment for patients with less melanin. ${ }^{1}$ Additionally, ultrasound technology does not cause enough edema or erythema to interfere with subsequent laser applications and thus offers an attractive adjunct for the treatment of neck laxity. ${ }^{43}$ In 60 patients treated initially with microfocused ultrasound with visualization (Ulthera System, Ulthera, Inc, Mesa, Arizona) using the 4.5-mm, 4-MHz; 3-mm, 7-MHz; and 1.5-mm, 10-MHz transducers at the highest energy settings delivering the number of lines based on manufacturer protocol followed immediately with treatment by an ablative fractionated laser, the improvement in skin laxity and texture of the neck was thought to be greater than the physicians' prior experience using either modality alone. The recovery time was delayed an additional 3 to 4 days, though the incidence in other adverse events was not noted. However, this article did not specify the skin types of the patients studied. ${ }^{44}$ Yet, when MFU-V was studied in 52 patients with skin types III-IV for tightening of the skin of the face and neck, the only prolonged adverse event noted was erythema in 1 patient. $^{45}$

If a patient wishes to address both pigmentary change in addition to skin tightening, Vanaman et al recommend beginning with a vascular laser, either IPL or PDL, then using tumescent anesthesia prior to subsurface monopolar RF (ThermiTight, ThermiAesthetics, Irving, Texas), and completing the session with a QS 532-, 694-, or 755-nm laser for any remaining pigmented lesions. As the 3 devices all have very different targets, no increased scarring or adverse events were noted. ${ }^{43}$

\section{Body}

\section{Cellulite}

Though affecting up to $80 \%$ of postpubertal women, cellulite remains a very difficult condition to treat, particularly using noninvasive technologies alone. ${ }^{46,47}$ However, the combination of mechanical manipulation, infrared light, and RF does show promise. Seven patients with skin phototypes ranging from III-V were treated with an RF light-based device (VelaSmooth, Syneron Medcial Ltd, Yokneam, Israel) that incorporates bipolar RF, infrared heat energy, and pulsatile vacuum suction through a handheld applicator. Following 8-9 treatments administered twice weekly, patients experienced an average of $25 \%$ improvement of cellulite on the abdomen and $50 \%$ on the thighs. ${ }^{48}$ The only adverse event reported was a single blister, which healed without scarring. Though the improvement was moderate at best, combination therapy using heat and mechanical stimulation does appear to be effective and safe for the treatment of cellulite in pigmented patients.

More recently, a subcision approach (Cellfina System; Ulthera Inc, Mesa, Arizona) has been described that manually dissects the fibrous network causing dimpling in the dermis. A precise depth of dissection may be fixed through a vacuum chamber to lift the tissue for the needle. As the Cellfina is a non-energy-based device, it is an attractive treatment option for patients with pigmented skin. Seventy-eight percent of patients enrolled in the original trial were skin type III and above, and 33\% of patients had skin type IV or above. Every subject noted an improvement at 1 year, and no longterm adverse events or PIH were recorded. ${ }^{49}$

\section{Scars}

Hypertrophic scars and keloids are quite common in skin of color patients and represent an exuberant healing response to trauma, inflammation, or burns. ${ }^{50}$ In addition to psychological distress caused by the appearance, keloids may also provoke sensations of pruritis or pain. ${ }^{51}$ Though numerous treatments have been proposed to improve the appearance and symptoms, keloids are notoriously recalcitrant or slow to respond to more conservative treatments such as intralesional steroid injections. Additionally, dyspigmentation and atrophy often accompany any improvement in the texture of the keloid. Unfortunately, keloids often recur, occasionally even growing to a larger size, after more aggressive or destructive techniques.

By using a combination approach with a 578-nm copper bromide laser to target the vascular component of the scar in addition to intralesional triamcinolone acetonide, keloids were noted to improve by at least $50 \%$ in 9 of 12 treated Korean patients when assessed by 2 physicians blinded to the treatment protocol. ${ }^{52}$ The increased improvement over injections alone was hypothesized to occur secondary to thermal injury to the scar microvasculature, which then induced ischemia and collagen reduction. ${ }^{52}$ The authors also noted significantly less purpura following the laser treatments than would be expected to occur when using a PDL.

Yan et al specifically studied 151 symptomatic keloids, which had been present for greater than 2 years, were greater than $2 \mathrm{~cm}^{2}$ in size, and had failed treatment with 2 or more treatment modalities, including excision, laser, cryotherapy, pressure garments, and radiation. Keloids were first treated with continuous-wave $\mathrm{CO} 2$ laser followed by ultra-pulse $\mathrm{CO} 2$ laser 5-7 days later in order to prevent the proliferation of scar tissue. Following the second $\mathrm{CO} 2$ treatment, ${ }^{24}$ P-patch contact brachyradiotherapy was performed. One hundred and eleven of the keloids responded without a noticeable scar or a cosmetically satisfactory scar following treatment, and 40 of the keloids resolved into a hypertrophied scar. None of the keloids were rated as being as large or larger than baseline following treatment, though approximately 50 treated sites were notable for residual hyperpigmentation or depigmentation. ${ }^{53}$ Thus, for particularly recalcitrant keloids, a combination therapy using both laser and radiation may provide significant benefit.

\section{Striae}

Striae distensae is a common cause of cosmetic concern characterized histologically by thinning of the epidermis as well as dermal collagen and elastin. ${ }^{54}$ If the striae are erythematous, the pigment may be treated safely in individuals with pigmented skin with a 
585-nm dye laser, though the textural change is much more difficult to improve..$^{55}$ More recently, a microneedle RF device (INTRAcel, Jeisys, Seoul, South Korea) has been shown to improve numerous dermal abnormalities such as rhytides, scars, and keloids. The textural improvement is thought to occur through wound remodeling and tightening in response to heat-induced collagen denaturation. ${ }^{56}$ With the combination of both RF technology (Thermage, Solta Medical, Inc, Hayward, California) and a 585-nm PDL, almost $90 \%$ of Asian patients noted an overall improvement in their striae, with approximately $60 \%$ showing "good or very good" improvement in the elasticity of their striae. ${ }^{57}$ In 10 patients with skin type $\mathrm{IV}$, the combination of fractional $\mathrm{CO} 2$ laser with microneedle RF (Secret; ilooda, Suwon, South Korea) showed greater improvement in their striae than 20 patients treated with either fractional CO2 laser or microneedle RF alone, with transient PIH occurring in $30 \%$ of patients in the combination group versus $20 \%$ in each of the other 2 groups. ${ }^{54}$ Thus, combination treatment was shown to be more effective without significant increase in adverse events in treatment of striae.

At our site, we have an ongoing split-body comparison trial to evaluate the efficacy of a nonablative fractionated 1565-nm laser (ResurFX, Lumenis Ltd, Santa Clara, California) and combination 1064-/532-nm fractionated picosecond laser (PicoWay Resolve, Syneron Candela, Irvine, California) in the treatment of striae. Skin types I-IV have been treated. Subjects receive 3 treatments spaced 3 weeks apart. Preliminary data reveal comparable efficacy between the laser treatments; however, there is more discomfort associated with the 1565-nm laser. In addition, PIH lasting longer than 1 month has been observed in the majority of subjects on the sides treated with the 1565-nm wavelength, whereas long-lasting $\mathrm{PIH}$ has not been appreciated on the sides randomized to the picosecond laser. Final data will be available later this year.

\section{Conclusion}

With each year, new medical, injectable, surgical, and device-based therapies, used alone or in combination, are reported in the pursuit of treating skin of color safely and successfully. The efficacy of combined regimens ("mega-combinations") is gaining popularity and is likely the future of treating most skin conditions to achieve optimal cosmesis. Additional research of combination treatment is necessary to document safety, to quantify the benefits, to establish the duration of effects, to review cost-effectiveness, and to evaluate the psychological improvement and quality of life for patients using the various treatment modalities.

\section{References}

1. Alexis AF. Lasers and light-based therapies in ethnic skin: treatment options and recommendations for Fitzpatrick skin types V and VI. Br J Dermatol. 2013;169(Suppl 3):91-97. doi:10.1111/bjd.12526.

2. Haimovic A, Brauer JA, Cindy Bae YS, Geronemus RG. Safety of a picosecond laser with diffractive lens array (DLA) in the treatment of Fitzpatrick skin types IV to VI: A retrospective review. J Am Acad Dermatol. 2016;74(5):931-936. doi:10.1016/j. jaad.2015.12.010.

3. Olson RL, Gaylor J, Everett MA. Skin color, melanin, and erythema. Arch Dermatol. 1973;108(4):541-544. doi:10.1001/archderm.1973.01620250029008.

4. Ross EV, Cooke LM, Overstreet KA, Buttolph GD, Blair MA. Treatment of pseudofolliculitis barbae in very dark skin with a long pulse Nd:YAG laser. J Natl Med Assoc. 2002;94:888-893.

5. Nanni CA, Alster TS. Complications of cutaneous laser surgery. A review. Dermatol Surg. 1998;24:209-219. doi:10.1016/S1076-0512(97)00286-0.

6. Rodrigues M, Pandya AG. Melasma: clinical diagnosis and management options. Australas J Dermatol. 2015;56(3):151-163. doi:10.1111/ajd.12290.
7. Angsuwarangsee S, Polnikorn N. Combined ultrapulse CO2 laser and Q-switched alexandrite laser compared with Q-switched alexandrite laser alone for refractory melasma: split-face design. Dermatol Surg. 2003;29(1):59-64. doi:10.1046/j.15244725.2003.29009.x.

8. Nouri K, Bowes L, Chartier T, Romagosa R, Spencer J. Combination treatment of melasma with pulsed $\mathrm{CO} 2$ laser followed by Q-switched alexandrite laser: a pilot study. Dermatol Surg. 1999;25(6):494-497. doi:10.1046/j.1524-4725.1999.08248.x.

9. Cunha PR, Pinto CA, Mattos CB, Cabrini DP, Tolosa JL. New insight in the treatment of refractory melasma: Laser Q-switched Nd: YAG non-ablative fractionated followed by intense pulsed light. Dermatol Ther. 2015;28(5):296-299. doi:10.1111/ dth. 12250 .

10. Wattanakrai P, Mornchan R, Eimpunth S. Low-fluence Q-switched neodymium-doped yttrium aluminum garnet $(1,064 \mathrm{~nm})$ laser for the treatment of facial melasma in Asians. Dermatol Surg. 2010;36(1):76-87. doi:10.1111/j.15244725.2009.01383.x.

11. Chan NP, Ho SG, Shek SY, Yeung CK, Chan HH. A case series of facial depigmentation associated with low fluence Q-switched 1,064 nm Nd:YAG laser for skin rejuvenation and melasma. Lasers Surg Med. 2010;42(8):712-719. doi:10.1002/ $1 \mathrm{sm} .20956$.

12. Vachiramon V, Sirithanabadeekul P, Sahawatwong S. Low-fluence Q-switched Nd: YAG 1064-nm laser and intense pulsed light for the treatment of melasma. $J$ Eur Acad Dermatol Venereol. 2015;29(7):1339-1346. doi:10.1111/jdv.12854.

13. Kauvar AN. Successful treatment of melasma using a combination of microdermabrasion and Q-switched Nd:YAG lasers. Lasers Surg Med. 2012;44(2):117-124. doi:10.1002/lsm.21156.

14. Fabi SG, Friedmann DP, Niwa Massaki AB, Goldman MP. A randomized, split-face clinical trial of low-fluence Q-switched neodymium-doped yttrium aluminum garnet $(1,064 \mathrm{~nm})$ laser versus low-fluence Q-switched alexandrite laser $(755 \mathrm{~nm})$ for the treatment of facial melasma. Lasers Surg Med. 2014;46(7):531-537. doi:10.1002/ $1 \mathrm{sm} .22263$

15. Vachiramon V, Sahawatwong S, Sirithanabadeekul P. Treatment of melasma in men with low-fluence Q-switched neodymium-doped yttrium-aluminum-garnet laser versus combined laser and glycolic acid peeling. Dermatol Surg. 2015;41(4):457465. doi:10.1097/DSS.0000000000000304

16. Chan HH, Leung RS, Ying SY, et al. A retrospective analysis of complications in the treatment of nevus of Ota with the Q-switched alexandrite and Q-switched Nd:YAG lasers. Dermatol Surg. 2000;26(11):1000-1006. doi:10.1046/j.15244725.2000.0260111000.x.

17. Watanabe S, Takahashi H. Treatment of nevus of Ota with the Q-switched ruby laser. N Engl J Med. 1994;331(26):1745-1750. doi:10.1056/NEJM199412293312604.

18. Alster TS, Williams CM. Treatment of nevus of Ota by the Q-switched alexandrite laser. Dermatol Surg. 1995;21(7):592-596. doi:10.1111/j.1524-4725.1995. tb00512.x

19. Tse Y, Levine VJ, McClain SA, Ashinoff R. The removal of cutaneous pigmented lesions with the Q-switched ruby laser and the Q-switched neodymium: yttrium-aluminum-garnet laser. A comparative study. J Dermatol Surg Oncol. 1994;20(12):795800. doi:10.1111/j.1524-4725.1994.tb03707.x.

20. Lowe NJ, Wieder JM, Sawcer D, Burrows P, Chalet M. Nevus of Ota: treatment with high energy fluences of the Q-switched ruby laser. J Am Acad Dermatol. 1993;29(6):997-1001. doi:10.1016/0190-9622(93)70280-7.

21. Park JM, Tsao H, Tsao S. Combined use of intense pulsed light and Q-switched ruby laser for complex dyspigmentation among Asian patients. Lasers Surg Med. 2008;40(2):128-133. doi:10.1002/lsm.20603.

22. Sakamoto FH, Lopes JD, Anderson RR. Photodynamic therapy for acne vulgaris: a critical review from basics to clinical practice: part I. Acne vulgaris: when and why consider photodynamic therapy? J Am Acad Dermatol. 2010;63(2):183-193; quiz 193-194. doi:10.1016/j.jaad.2009.09.056.

23. Yin R, Hao F, Deng J, Yang XC, Yan H. Investigation of optimal aminolaevulinic acid concentration applied in topical aminolaevulinic acid-photodynamic therapy for treatment of moderate to severe acne: a pilot study in Chinese subjects. $\mathrm{Br} \mathrm{J}$ Dermatol. 2010;163(5):1064-1071. doi:10.1111/j.1365-2133.2010.09860.x.

24. Yin R, Lin L, Xiao Y, Hao F, Hamblin MR. Combination ALA-PDT and ablative fractional Er:YAG laser $(2,940 \mathrm{~nm})$ on the treatment of severe acne. Lasers Surg Med. 2014;46(3):165-172. doi:10.1002/lsm.22219.

25. Sadick NS, Krueger N. Advances in Cosmetic Dermatology. Philadelphia, Pennsylvania: Elsevier Inc; 2014

26. Munavalli G. Single-Center, Prospective Study on the Efficacy and Safety of Microfocused Ultrasound with Visualization for the Non-invasive Treatment of Moderate to Severe Facial Acne. American Society for Laser Medicine and Surgery Conference. Boston, MA. April, 2013.

27. Joseph J. Prospective, Multi-Site, Initial Pilot Investigation of the Safety and Effectiveness of Micro-Focused Ultrasound with Visualization for Correction of Moderate to Severe Atrophic Acne Scars. American Academy of Facial Plastic and Reconstructive Surgery; 2015; Boston, MA.

28. Wang B, Wu Y, Luo YJ, et al. Combination of intense pulsed light and fractional 
$\mathrm{CO}(2)$ laser treatments for patients with acne with inflammatory and scarring lesions. Clin Exp Dermatol. 2013;38(4):344-351. doi:10.1111/ced.12010.

29. Saedi N, Jalian HR, Petelin A, Zachary C. Fractionation: past, present, future. Semin Cutan Med Surg. 2012;31(2):105-109. doi:10.1016/j.sder.2012.02.003.

30. Metelitsa AI, Alster TS. Fractionated laser skin resurfacing treatment complications: a review. Dermatol Surg. 2010;36(3):299-306. doi:10.1111/j.15244725.2009.01434.x.

31. Simmons BJ, Bray FN, Falto-Aizpurua LA, Nouri K. The use of radiofrequency in combination with lasers for acne scars. Int J Dermatol. 2016;55(5):e312-e315. doi:10.1111/ijd.13106.

32. Peterson JD, Palm MD, Kiripolsky MG, Guiha IC, Goldman MP. Evaluation of the effect of fractional laser with radiofrequency and fractionated radiofrequency on the improvement of acne scars. Dermatol Surg. 2011;37(9):1260-1267. doi:10.1111/ j.1524-4725.2011.02110.x.

33. Taub AF, Garretson CB. Treatment of acne scars of skin types II to V by sublative fractional bipolar radiofrequency and bipolar radiofrequency combined with diode laser. J Clin Aesthet Dermatol. 2011;4(10):18-27.

34. Ibrahimi OA, Weiss RA, Weiss MA, et al. Treatment of acne scars with high intensity focused radio frequency. J Drugs Dermatol. 2015;14(9):1065-1068.

35. Zhou BR, Xu Y, Guo SL, et al. The effect of conditioned media of adipose-derived stem cells on wound healing after ablative fractional carbon dioxide laser resurfacing. Biomed Res Int. 2013;2013:519126. doi:10.1155/2013/519126.

36. Kim WS, Park BS, Sung JH, et al. Wound healing effect of adipose-derived stem cells: a critical role of secretory factors on human dermal fibroblasts. $J$ Dermatol Sci. 2007;48(1):15-24. doi:10.1016/j.jdermsci.2007.05.018.

37. Zhou BR, Zhang T, Bin Jameel AA, et al. The efficacy of conditioned media of adipose-derived stem cells combined with ablative carbon dioxide fractional resurfacing for atrophic acne scars and skin rejuvenation. J Cosmet Laser Ther. 2016;18(3):138-148. doi:10.3109/14764172.2015.1114638.

38. Sadick NS, Makino Y. Selective electro-thermolysis in aesthetic medicine: a review. Lasers Surg Med. 2004;34(2):91-97. doi:10.1002/lsm.20013.

39. Sadick NS, Trelles MA. Nonablative wrinkle treatment of the face and neck using a combined diode laser and radiofrequency technology. Dermatol Surg. 2005;31(12):1695-1699. doi:10.2310/6350.2005.31310.

40. Tanaka Y, Tsunemi Y, Kawashima M, Tatewaki N, Nishida H. Objective assessment of skin tightening in Asians using a water-filtered near-infrared (1,000-1,800 nm) device with contact-cooling and freezer-stored gel. Clin Cosmet Investig Dermatol. 2013;6:167-176. doi:10.2147/CCID.S47299.

41. Tierney EP, Hanke CW. Ablative fractionated CO2, laser resurfacing for the neck: prospective study and review of the literature. J Drugs Dermatol. 2009;8(8):723-731.

42. Jerdan K, Fabi S. A noninvasive approach to off-face skin laxity and tightening: a review of the literature. Semin Cutan Med Surg. 2015;34(3):118-128. doi:10.12788/j. sder.2015.0173.

43. Vanaman M, Fabi SG, Cox SE. Neck Rejuvenation using a combination approach: our experience and a review of the literature. Dermatol Surg. 2016;42(Suppl 2):S94S100. doi:10.1097/DSS.0000000000000699.

44. Woodward JA, Fabi SG, Alster T, Colon-Acevedo B. Safety and efficacy of combining microfocused ultrasound with fractional $\mathrm{CO} 2$ laser resurfacing for lifting and tightening the face and neck. Dermatol Surg. 2014;40 Suppl 12:S190-S193. doi:10.1097/DSS.0000000000000228.

45. Harris MO, Sundaram HA. Safety of microfocused ultrasound with visualization in patients with Fitzpatrick skin phototypes III to VI. JAMA Facial Plast Surg. 2015;17(5):355-357. doi:10.1001/jamafacial.2015.0990.

46. Rossi AB, Vergnanini AL. Cellulite: a review. J Eur Acad Dermatol Venereol. 2000;14(4):251-262. doi:10.1046/j.1468-3083.2000.00016.x.

47. Goldman MP. Cellulite: a review of current treatments. Cosmet Dermatol. 2002; $15: 17-20$

48. Wanitphakdeedecha R, Manuskiatti W. Treatment of cellulite with a bipolar radiofrequency, infrared heat, and pulsatile suction device: a pilot study. $J$ Cosmet Dermatol. 2006;5(4):284-288. doi:10.1111/j.1473-2165.2006.00271.x.

49. Kaminer MS, Coleman WP III, Weiss RA, Robinson DM, Coleman WP IV, Hornfeldt C. Multicenter pivotal study of vacuum-assisted precise tissue release for the treatment of cellulite. Dermatol Surg. 2015;41(3):336-347. doi:10.1097/ DSS.0000000000000280.

50. English RS, Shenefelt PD. Keloids and hypertrophic scars. Dermatol Surg. 1999;25(8):631-638. doi:10.1046/j.1524-4725.1999.98257.x

51. Bock O, Schmid-Ott G, Malewski P, Mrowietz U. Quality of life of patients with keloid and hypertrophic scarring. Arch Dermatol Res. 2006;297(10):433-438 doi:10.1007/s00403-006-0651-7.

52. Son IP, Park KY, Kim B, Kim MN. Pilot Study of the Efficacy of $578 \mathrm{~nm}$ Copper Bromide Laser Combined with Intralesional Corticosteroid Injection for Treatment of Keloids and Hypertrophic Scars. Ann Dermatol. 2014;26(2):156-161. doi:10.5021/ad.2014.26.2.156.

53. Yan D, Zhao B, Yang H, Zhu B, Wang J. A combination of nonoperative treatment modalities used for treatment of keloids. Dermatol Ther. 2014;27(1):48-51. doi:10.1111/dth. 12044

54. Ryu HW, Kim SA, Jung HR, Ryoo YW, Lee KS, Cho JW. Clinical improvement of striae distensae in Korean patients using a combination of fractionated microneedle radiofrequency and fractional carbon dioxide laser. Dermatol Surg. 2013:39(10):1452-1458. doi:10.1111/dsu.12268.

55. Jiménez GP, Flores F, Berman B, Gunja-Smith Z. Treatment of striae rubra and striae alba with the 585-nm pulsed-dye laser. Dermatol Surg. 2003;29(4):362-365. doi:10.1046/j.1524-4725.2003.29086.x.

56. Pritzker RN, Hamilton HK, Dover JS. Comparison of different technologies for noninvasive skin tightening. J Cosmet Dermatol. 2014;13(4):315-323. doi:10.1111/jocd.12114.

57. Suh DH, Chang KY, Son HC, Ryu JH, Lee SJ, Song KY. Radiofrequency and 585 $\mathrm{nm}$ pulsed dye laser treatment of striae distensae: a report of 37 Asian patients. Dermatol Surg. 2007;33(1):29-34. doi:10.1111/j.1524-4725.2007.33004.x. 